\title{
Simulations of Crystallization in Supercooled Nanodroplets in the Presence of a Strong Exothermic Solute
}

\author{
Søren Toxvaerd, ${ }^{*,+}$ Niels Larsen, ${ }^{\ddagger}$ and Jeppe C. Dyre ${ }^{\dagger}$ \\ ${ }^{\dagger}$ DNRF centre “Glass and Time”, Department of Sciences, Roskilde University, Postbox 260, DK-4000 Roskilde, Denmark \\ ${ }^{\ddagger}$ Danish Meteorological Institute, Lyngbyvej 100, DK-2100 Copenhagen, Denmark
}

\begin{abstract}
Molecular dynamics simulations of crystallization in supercooled nanodroplets of binary Lennard-Jones mixtures with a solvent and a strong exothermic solute corresponding to a sulfuric acid aerosol demonstrate that the presence of the solute controls the nucleation. A strongly exothermic solute prefers to be surrounded at all sides by solvent molecules, and for this reason the outermost $1-2$ surface layers are depleted of solute and with a diluted concentration of the solvent. The crystallization is observed to initiate a few layers beneath the surface and to result in a crystal growth that encapsulates the droplet by an "egg shell" of a crystal of solvent particles. Our findings are discussed in relation to experimental data of ice formation in polar stratospheric clouds.
\end{abstract}

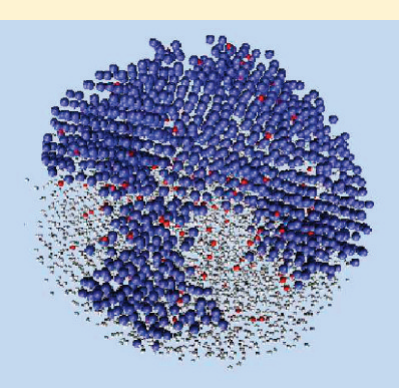

\section{INTRODUCTION}

The vitrification and crystallization of nanodroplets is a subject of great interest. ${ }^{1}$ In the article, we examine, by Molecular Dynamics (MD) simulations, for the first time the onset of nucleation and crystal growth in supercooled nanodroplets in the presence of a strong exothermic solute.

MD simulations offer the possibility to identify and locate the critical crystal nucleus. Crystallization by simulations of small droplets of $\mathrm{SeF}_{6}$ was first obtained by Chushak and Bartell. ${ }^{2,3}$ They observed that crystallization appeared spontaneously near the surface of the droplets. In a recent extensive simulation of crystallization in a binary mixture of $\mathrm{Si}$ and $\mathrm{Ge},{ }^{4}$ the authors observed "that the nucleation does not occur exactly at the surface but instead in a subsurface region that is a few atomic layers underneath". This fact was explained as caused by a negative slope of the melting line, a rare feature in general but consistent with the melting line of $\mathrm{H}_{2} \mathrm{O}$. A MD simulation of the density fluctuations in water with free surfaces ${ }^{5}$ indicated that the ice-ordering appeared near the surface, and in a series of papers Djikaev et al. ${ }^{6-8}$ developed a theory for the thermodynamic conditions for surface-stimulated crystallization. According to their theory, the onset of crystallization and the subsequent growth appear near ${ }^{8}$ or at the surface, provided at least one crystal facet is only partially wettable by its own melt. Here we observe by MD surface-induced onset of crystallization and crystal growth in nanodroplets of mixtures of solvent molecules and solute molecules with a strong negative mixing enthalpy. We conclude that several factors might support surfaceinduced crystallization in water, as well as in other systems.

Many simulations of supercooled highly viscous fluids reported in the literature were performed for the Kob-Andersen binary Lennard-Jones mixture (KABLJ), ${ }^{9}$ which is resistant against crystallization due to a large negative enthalpy of mixing. ${ }^{10}$ An exothermic mixture not only increases the melting-point depression significantly but also suppresses the composition fluctuations necessary for a critical nucleus of the solvent molecules to appear. ${ }^{10}$ We have determined the crystallization in nanodroplets for the original KABLJ mixture, as well as for a modified KABLJ model of a sulfuric acid aerosol. There are no qualitative differences in the onset of nucleations and crystal growth between the two systems. Below we report the results obtained for the modified KABLJ droplets.

The article is organized as follow: Section II A outlines the KABLJ model for the mixture with a large negative enthalpy of mixing, Section II B contains thermodynamic data for sulfuric acid aerosols in the upper troposphere and lower stratosphere (UTLS), and in Section II C we determine the values of the potential parameters in the model for a sulfuric acid aerosol. Section III describes the MD simulations of supercooled nanodroplets. Section IV shows the crystallization and crystal growth in the nanodroplets. In Section V we summarize the results in the context of experiments studying crystallization in droplets, including sulfuric acid aerosols. The perspective of our results for ice formation in polar stratospheric clouds is also briefly discussed.

\section{KABLJ MODELS FOR SUPERCOOLED NANODROPLETS}

A. LJ Mixture with Negative Mixing Enthalpy. The stability of a supercooled binary liquid mixture against crystallization of the solvent molecules depends strongly on the enthalpy of mixing, $\Delta_{\text {mix }} H .^{10}$ The KABLJ mixture is resistant against crystallization due to its large negative enthalpy of mixing. The exothermic strength of the KABLJ mixtures was in ref 10 monitored by scaling the interactions between the solute (A) particles

Received: March 22, 2011

Revised: June 1, 2011

Published: June 02, 2011 
and the solvent (B) particles, whereby the potential parameters deviate considerably from the "ideal-mixture" Lorentz-Berthelot (LB) rules for binary mixtures ${ }^{11}$

$$
\begin{gathered}
\sigma_{\mathrm{AB}}=\left(\sigma_{\mathrm{AA}}+\sigma_{\mathrm{BB}}\right) / 2 \\
\varepsilon_{\mathrm{AB}}=\sqrt{\varepsilon_{\mathrm{AA}} \varepsilon_{\mathrm{BB}}}
\end{gathered}
$$

Here $\sigma_{\alpha \beta}$ is the diameter, and $\varepsilon_{\alpha \beta}$ is the energy strength of the pair potential, $\alpha, \beta \in[\mathrm{A}, \mathrm{B}])$. We use standard Lennard-Jones (LJ) potentials

$$
u\left(r_{i j}\right)=4 \varepsilon_{\alpha \beta}\left[\left(\frac{\sigma_{\alpha \beta}}{r_{i j}}\right)^{12}-\left(\frac{\sigma_{\alpha \beta}}{r_{i j}}\right)^{6}\right]
$$

for the potential energy between molecules $i$ and $j$ at the distance $r_{i j}$ (the pair potentials are truncated at $r_{\alpha \beta}=2.5 \sigma_{\alpha \beta}$ ).

The strength of the exothermic mixtures, given by the enthalpy of mixing $\Delta_{\text {mix }} H$, depends on the relative deviation from the LB energy mixing rule. We define the following dimensionless measure of the exothermic strength in the KABLJ model

$$
\Delta \equiv \frac{\varepsilon_{\mathrm{AB}}}{\sqrt{\varepsilon_{\mathrm{AA}} \varepsilon_{\mathrm{BB}}}}
$$

The exothermic strength $\Delta$ is larger than unity for an exothermic mixture. It increases the strength at which the solute binds to the solvent, whereby crystallization of the pure solvent by phase separation is suppressed. ${ }^{10}$ Crystallization of the solvent in supercooled systems depends strongly on the strength of the exothermic mixture. In ref 10 the exothermic strength for a KABLJ mixture was varied from $\Delta=1.32$ to 2.12 , corresponding to a change in $\Delta_{\text {mix }} H$ from -3 to $-8 \mathrm{~kJ} / \mathrm{mol}$, by which the mean crystallization time for the bulk system increased by a factor of roughly 200.

B. Sulfuric Acid Aerosols in UTLS. Aerosols and cloud particles in the upper troposphere and lower stratosphere play an important role in the radiative balance of the climate system, ${ }^{12}$ influence the transport of water vapor to the stratosphere in the tropical tropopause layer through microphysical processes, ${ }^{13}$ and provide surfaces for heterogeneous chemical reactions in the ozone layer. ${ }^{14}$ The creation of crystals is initiated by nanometersized aerosols, which serve as seeds for the nucleation and freezing of $\mathrm{H}_{2} \mathrm{O}$ and nitric acid trihydrate (NAT). Some aerosols are glassy or highly viscous droplets, and generally aerosols contain sulfuric as well as nitric acid molecules. Homogeneous ice nucleation depends only indirectly on the chemical nature of the solute; it is controlled by the activity of water in the aerosol. ${ }^{15}$

Aerosols contain water (A), sulfuric (B), nitric acid, and hydrates. ${ }^{16-18}$ The phase diagrams for just binary mixtures with these hydrates are very complex; in the case of water and sulfuric acid, there are no less than four eutectic points between crystal hydrates $\mathrm{H}_{2} \mathrm{SO}_{4} \cdot n \mathrm{H}_{2} \mathrm{O}$ with $1 \leq n \leq 8 .{ }^{19,20}$ The eutectic temperature in the dilute regime between pure ice and sulfuric acid tetrahydrate is depressed to $199.32 \pm 0.43 \mathrm{~K}$ at a weight percent of roughly 40 of $\mathrm{H}_{2} \mathrm{SO}_{4}$, corresponding to a mole fraction of sulfuric acids of $x_{\mathrm{B}}=0.12$. The mixture of $\mathrm{H}_{2} \mathrm{O}$ and $\mathrm{H}_{2} \mathrm{SO}_{4}$ is strongly nonideal with a large negative enthalpy of mixing, $\Delta_{\text {mix }} H$, with a minimum in $\Delta_{\text {mix }} H=-14.395 \mathrm{~kJ} / \mathrm{mol}$ at $x_{\mathrm{H}_{2} \mathrm{SO}_{4}}=0.42 .{ }^{21-23}$ The presence of nitric acid in a ternary mixture further lowers the eutectic point to $197.4 \mathrm{~K}^{24}$ Sulfuric acid is the dominating exothermic component in the complex mixture. This explains the significant freezing-point depression of ice in the aerosols for mole fractions $x_{\mathrm{S}}<0.12$ (corresponding to a molal concentration $\left.m\left(\mathrm{H}_{2} \mathrm{SO}_{4}\right)=7.57\right)$. The diluted mixture $\left(x_{\mathrm{H}_{2} \mathrm{SO}_{4}} \ll 0.12\right)$ of $\mathrm{H}_{2} \mathrm{O}(1)$ and $\mathrm{H}_{2} \mathrm{SO}_{4}(\mathrm{l})$ in the supercooled aerosols consists of $\mathrm{H}_{3} \mathrm{O}^{+}(\mathrm{aq})$ and $\mathrm{SO}_{4}{ }^{2-}$ (aq). The sulfuric anion has a perfect tetrahedral structure. The $\mathrm{S}-\mathrm{O}^{-\delta}$ bond length is shorter than the corresponding $\mathrm{S}-\mathrm{O}-\mathrm{H}$ bond length, and the ion is compact and spherical-like. ${ }^{25}$

C. KABLJ Model for a Sulfuric Acid Aerosol. The parameters $\sigma_{\alpha, \beta}$ and $\varepsilon_{\alpha, \beta}$ for the KABLJ model of an exothermic mixture of $\mathrm{H}_{2} \mathrm{O}(\mathrm{l})$ and $\mathrm{H}_{2} \mathrm{SO}_{4}(\mathrm{l})$ were determined from physical-chemical data for $\mathrm{H}_{2} \mathrm{O}, \mathrm{H}_{2} \mathrm{SO}_{4}$, and $\mathrm{H}_{2} \mathrm{SO}_{4}$ (aq) by proceeding as follows: The melting-point temperature of $\mathrm{H}_{2} \mathrm{SO}_{4}(\mathrm{c})$ is $T_{\text {fus }}=283.5 \mathrm{~K}^{26}$ The fact that the melting-point temperature of $\mathrm{H}_{2} \mathrm{SO}_{4}(\mathrm{c})$ approximately equals the corresponding melting-point temperature of ice means that the effective LJ energy parameters for the one-component systems of $\mathrm{H}_{2} \mathrm{O}$ and of $\mathrm{H}_{2} \mathrm{SO}_{4}$ must be almost identical, $\varepsilon_{\mathrm{BB}} \approx \varepsilon_{\mathrm{AA}}$, since they have the same LJ triplepoint temperature, $T_{\mathrm{t}}$, and thereby the same reduced temperature $k_{\mathrm{B}} T_{\mathrm{t}} / \varepsilon$. The triple-point temperature of $\mathrm{H}_{2} \mathrm{O}$ is $273.16 \mathrm{~K}$, and the triple-point temperature and liquid density of the (truncated) LJ system is $k_{\mathrm{B}} T / \varepsilon_{\mathrm{AA}}=0.618$ and $\rho \sigma_{\mathrm{AA}}^{3}=$ $0.8290 .^{27}$ This gives $\varepsilon_{\mathrm{AA}} / k_{\mathrm{B}}=\varepsilon_{\mathrm{BB}} / k_{\mathrm{B}} \approx 442 \mathrm{~K}$ and $\sigma_{\mathrm{AA}}=$ $0.3104 \mathrm{~nm}$ (the value of $\varepsilon_{\mathrm{AA}} / k_{\mathrm{B}}$ is used to calculate $\Delta H_{\text {mix }}$ from $\left.\Delta u_{\text {mix }}\right)$. The densities of $\mathrm{H}_{2} \mathrm{O}(1)$ and $\mathrm{H}_{2} \mathrm{SO}_{4}(1)$ are 1.000 and $1.832 \mathrm{~g} / \mathrm{cm}^{3}$, respectively, which gives a ratio $\sigma_{\mathrm{BB}} / \sigma_{\mathrm{AA}}=1.44$. The mass ratio is $m_{\mathrm{B}} / m_{\mathrm{A}} \approx 5$ in the case of sulfuric acid or its ionics. The density of $\mathrm{H}_{2} \mathrm{SO}_{4}(\mathrm{aq})$ varies almost linearly with composition, ${ }^{28}$ which means that the simple $\mathrm{LB}$ rule, $\sigma_{\mathrm{AB}}=\left(\sigma_{\mathrm{AA}}+\right.$ $\left.\sigma_{\mathrm{BB}}\right) / 2=1.22$, gives the correct first-order description of the density dependence of $\mathrm{H}_{2} \mathrm{SO}_{4}(\mathrm{aq})$.

The exothermic strength of the mixture is controlled by the value of $\Delta$. There exist no experimental data for $\Delta H_{\text {mix }}$ in nanosized droplets at the relevant temperature of freezing $T \approx 180-$ $200 \mathrm{~K}$. The heat of mixing of sulfuric acid with water, $-\Delta H_{\text {mix }^{-}}$ $\left(x_{\mathrm{H}_{2} \mathrm{SO}_{4}}\right)$, at room temperature has a parabolic shape with a maximum at $x_{\mathrm{H}_{2} \mathrm{SO}_{4}}=0.42$ and the value $\approx 12 \mathrm{~kJ} / \mathrm{mol}$ for $x_{\mathrm{H}_{2} \mathrm{SO}_{4}}=$ $0.25 .^{23}$ For small concentrations, it follows Henry's law and varies linearly with the mole fraction and the concentration. ${ }^{29}$ The heat of mixing decreases with decreasing temperatures.

The strength of the exothermic mixture is taken to $\Delta=1.5$, i.e., $\varepsilon_{\mathrm{AB}}=1.5, \varepsilon_{\mathrm{AA}}$ which gives $\Delta u_{\mathrm{mix}} / \varepsilon_{\mathrm{AA}}=-0.9435$ at $k_{\mathrm{B}} T / \varepsilon_{\mathrm{AA}}=$ 0.40 for $x_{\mathrm{B}}=0.1$ in the KABLJ model for the sulfuric acid aerosol. This corresponds to the enthalpy of mixing $\Delta H_{\text {mix }} \approx-3.5 \mathrm{~kJ} /$ $\mathrm{mol}$ at this concentration (due to the very low vapor pressure one has $\left.\Delta H_{\text {mix }} \approx \Delta U_{\text {mix }}\right)$. The heat of mixing for the KABLJ model varies linearly with the concentration for small values of $x_{\mathrm{B}}$. We believe the value of $\Delta=1.5$ is a realistic value for a sulfuric acid aerosol, but in the absence of experimental data we also simulated droplets with other values. A higher value suppresses crystallization, whereas a smaller value enhances it; however, we observed no qualitative differences either in the density distributions in the supercooled droplets before crystallization or in the location of the crystal nucleus.

The exothermic strength of the mixture, $\Delta$, determines the ratio of the activities in the binary mixture. The absolute values of the chemical potentials and the activities of the components in the case of water with sulfuric acid can, however, not be obtained by the KABLJ model. To determine the absolute activity-and thereby the equilibrium vapor pressure of the solvent at supercooling-one needs the correct value of the enthalpy of sublimation, $\Delta H_{\text {sup }}(T)$, according to the Clausius-Clapeyron equation. For a solvent of $\mathrm{H}_{2} \mathrm{O}$ it can only be obtained by including 


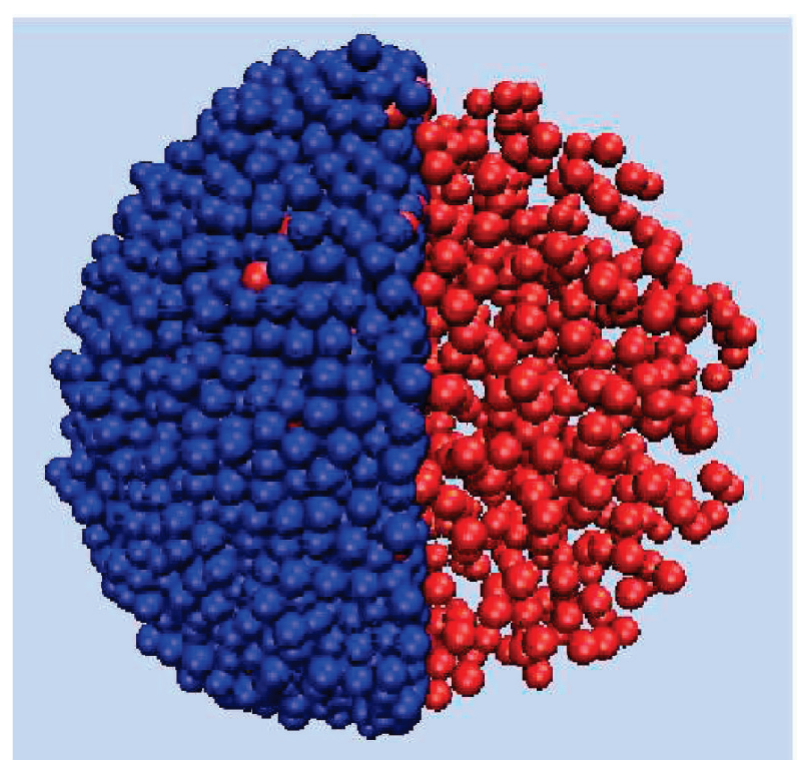

Figure 1. Side view of a supercooled nanodroplet with mole fraction $x_{\mathrm{B}}=0.1$ of the solute molecules (red). The solvent molecules (blue) encapsulate the (hydrophilic) solute molecules. Only the solute molecules are shown in the right half of the picture.

hydrogen-bond interactions: At the triple-point $T_{\mathrm{t}}$ one has $\Delta H_{\text {sup }}\left(T_{\mathrm{t}}\right)=T_{\mathrm{t}}\left[\Delta S_{\text {fus }}\left(T_{\mathrm{t}}\right)+\Delta S_{\text {vap }}\left(T_{\mathrm{t}}\right)\right]$, and both entropy terms depend strongly on the hydrogen bonds in solid and liquid $\mathrm{H}_{2} \mathrm{O}$.

A simple supercooled LJ droplet freezes spontaneously for $k_{\mathrm{B}} T / \varepsilon_{\mathrm{AA}} \leq 0.55,{ }^{27}$ but crystallization in supercooled droplets of strongly exothermic mixtures is suppressed too much lower temperatures. This is also the case for ice formation in supercooled stratospheric aerosols, for which the crystallization of $\mathrm{H}_{2} \mathrm{O}(\mathrm{c})$ takes place at temperatures $T \approx 180-200 \mathrm{~K},{ }^{30}$ corresponding to a supercooled temperature in the LJ droplets in the interval $k_{\mathrm{B}} T / \varepsilon_{\mathrm{AA}} \approx[0.40,0.45]$ with the values of the potential parameters given above.

\section{SUPERCOOLED NANODROPLET}

A. Molecular Dynamics Simulation of a Nanodroplet. The sizes of aerosols are typically in the range $10-100 \mathrm{~nm}$. A small droplet of $10 \mathrm{~nm}$ contains of the order of 10000 molecules which can be simulated with today's fastest computers over what corresponds to microseconds. This is sufficient to observe crystallization in supercooled, highly viscous mixtures. The system was set up in the following way: $N(\mathrm{~A})$ A particles and $N(\mathrm{~B}) \mathrm{B}$ particles $(N(\mathrm{~A})+N(\mathrm{~B})=10000)$ were placed in the center of a cubic box with edge length significantly longer than the diameter of the droplet. A repulsive potential at the box surfaces ${ }^{31}$ keeps the molecules in the box by reflecting the (few) molecules in the gas phase which surround the droplet. The system's center of mass was adjusted to the center of the box. Droplets were equilibrated at constant temperature, and the droplet momentum and angular momentum were adjusted to zero during the temperature equilibration and relaxation to the equilibrium state. ${ }^{32}$ After equilibration over typically millions of time steps (a time step is $\left.h=0.005 \sigma_{\mathrm{AA}}\left(m_{\mathrm{A}} / \varepsilon_{\mathrm{AA}}\right)^{1 / 2} \approx 6.3 \times 10^{-13} \mathrm{~s}\right)$, the systems contained few molecules outside the droplet, corresponding to a very low vapor pressure.

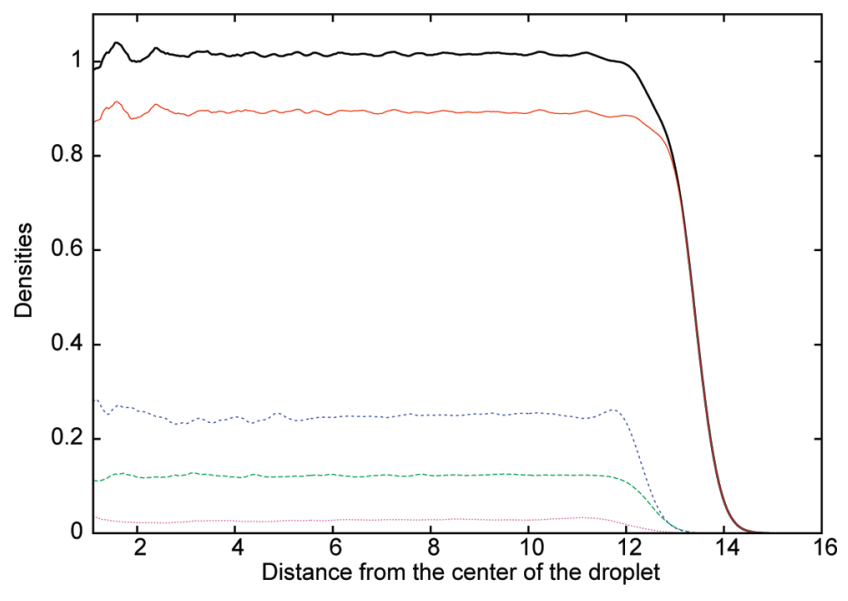

Figure 2. Densities, $\rho_{\mathrm{A}}(r)$ and $\rho_{\mathrm{B}}(r)$, of solvent $(\mathrm{A})$ and the exothermic solute $(\mathrm{B})$ in the supercooled nanodroplet as a function of the distance to the center of the droplet for various fractions of $B$ molecules (length unit: diameter $\left.\sigma_{\mathrm{AA}}=0.3104 \mathrm{~nm}\right)$. The red line gives $\rho_{\mathrm{A}}(r)$; green dashes give $\rho_{\mathrm{B}}(r)$; and the black line gives $\rho_{\mathrm{A}}(r)+\rho_{\mathrm{B}}(r)$, for the mixture with the stoichiometric fraction of $x_{\mathrm{B}}=0.1$. Blue dashes: $x_{\mathrm{B}}=0.2$. Magenta dots: $x_{\mathrm{B}}=0.0225$. The exothermic solvent and solute particles are uniformly distributed in the interior of the droplet, but the exothermic droplet is covered with a thin shell of solvent particles with a tanh decreasing density in the liquid-gas interface.

Most simulations were performed as constant-temperature MD. The algorithms and details are given in ref 32 . To locate the onset of crystallization and the growth of the critical nucleus without a substantial increase of computer time, we use a cluster routine which sorts $N$ objects within a computer time proportional to $N^{33}$

B. Supercooled Viscous Nanodroplet. The simulations of droplets of $10000 \mathrm{~A}$ and B particles show that the droplets remained in the supercooled state without any tendency to crystallize for particle fractions $x_{\mathrm{B}} \geq 0.025$ at $k_{\mathrm{B}} T / \varepsilon_{\mathrm{AA}}=0.40$ (corresponding to the temperature $T \approx 180 \mathrm{~K}$ where $\mathrm{H}_{2} \mathrm{O}$ in a sulfuric acid aerosol freezes $\left.{ }^{30}\right)$, even after very long time $\left(2 \times 10^{7}\right.$ time steps, corresponding to $10 \mu \mathrm{s})$. This resistance against crystallization is due to the exothermic strength of the mixture, which results in a higher mole fraction of the solute in the interior of the droplet, suppressing the onset of crystallization. ${ }^{10}$ A cut through the droplet is shown in Figure 1. In Figure 2 we show the densities of solvent and solute, $\rho_{\mathrm{A}}(r)$ and $\rho_{\mathrm{B}}(r)$, as functions of the distance, $r$, from the center of mass at $k_{\mathrm{B}} T / \varepsilon_{\mathrm{AA}}=0.40$ for various molecular fractions and for the exothermic strength $\Delta=1.5$. As is clear from the figures, there is in general an increased concentration of the solute B particles in the interior of the droplet and a depletion shell at the surface with a thickness of $1-2$ molecular diameters with a diluted concentration of pure solvent.

The density profiles in Figure 2 represent a central finding of this paper: Whenever a solute particle is exothermic, it becomes energetically favorable for it to surround itself by as many solvent particles as possible. This means that the solute concentration drops to zero at a distance from the surface 1-2 molecule layers before the solvent concentration. A liquid-gas interface is smooth with a tanh-decreasing density, ${ }^{34}$ and inspection of the densities in Figure 2 reveals that the solvent density, $\rho_{A}$, is part of this interface. Thus, the exothermic KABLJ droplet is covered by a thin shell of solvent particles with a tanh decreasing density. 


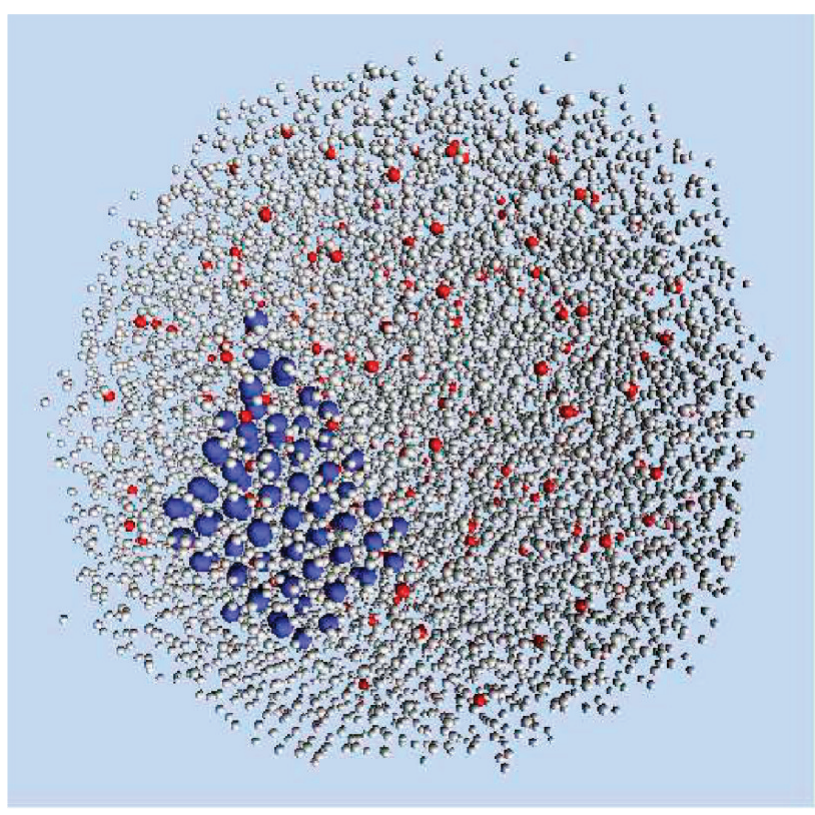

Figure 3. Detailed view of a droplet at the onset of crystallization. The solvent A particles (white) and solute B particles (red) are scaled down to show the (114) A particles in the crystal nucleus (blue) (defined as A12 particles where an A12 particle is as a A particle surrounded within a lattice distance by 12 other A particles).

The exothermic mixtures' resistance against crystallization is caused by several factors. The increased solute concentration in the interior of the droplet enhances the freezing-point depression and requires an (exponentially) less likely molecular fluctuation, necessary for the creation of a critical crystal nucleus of pure A particles. ${ }^{10}$ The thin and diluted surface layer of the solvent does not crystallize due to the diluted concentration and because a quasi-two-dimensional (2D) layer of solvent molecules is not inclined to crystallize. ${ }^{35}$ In fact, crystals are known to have melted surface layer(s). ${ }^{36}$ Ice has a liquid-like surface deep below the melting-point temperature; for a planar surface, ice has at least a liquid-like wetted layer for $T \geq 250 \mathrm{~K},{ }^{36}$ and the droplet's curvature further enhances this effect. Altogether, these factors explain why supercooled nanodroplets are so stable against crystallization. As we shall see, the same factors determine both the eventual onset of crystallization and the subsequent crystal growth in the droplets.

\section{CRYSTALLIZATION IN NANODROPLETS}

The main aim of our investigation is to determine where in a supercooled nanodroplet the critical crystal nucleus of solvent molecules is created and to determine the growth of the crystal. In computer simulations of nucleation and phase separation one identifies the critical nucleus as a cluster of particles in the new phase that can either return to the metastable phase or grow further with equal probability. ${ }^{37} \mathrm{MD}$ offers the possibility not only to identify this state but also to determine the kinetics ${ }^{38}$ and the growth of the new phase. ${ }^{39}$ For this purpose we need a local so-called order parameter, which changes at the onset of crystallization. The solvent in the KABLJ mixture freezes into a closepacked crystal structure (fcc or hcp), ${ }^{10}$ and as an order parameter we use the number of A particles that have 12 nearest A particles

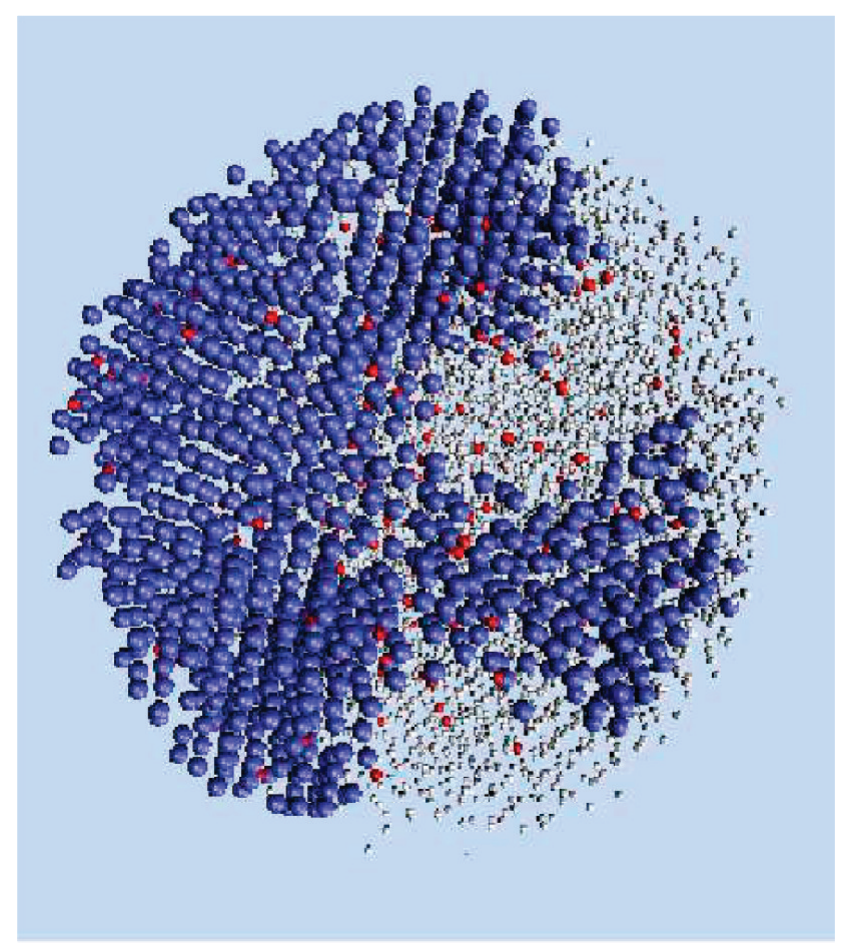

Figure 4. Later stage of crystallization of the droplet shown in Figure 3 where the crystal nucleus contains 1340 particles.

within one closed-packed lattice distance $\left(1.25 \sigma_{\mathrm{AA}}\right)$ from them (such A particles are designated A12).

Before onset of crystallization, the A12 particles are randomly distributed within the uniform mixture in the interior of the droplet. At the onset of nucleation the number of A12 particles increases rapidly and monotonically, and the A12 particles tend to form clusters (defined by requiring that at least two A12 particles in a cluster are within one lattice distance from each other). The critical crystal is far from the compact spherical-like object usually assumed in classical nucleation theory. Rather, it is a quasi-two-dimensional thin crystal which grows in a shell just below the interior-interface zone, as one might expect from the fact that the concentration of $\mathrm{B}$ particles near the surface is low (Figure 2). In Figure 3 we show the critical crystal of A12 particles at the onset of crystallization, while Figure 4 shows the droplet after $5 \times 10^{6}$ time steps $(\approx 3 \mu \mathrm{s})$ after the onset.

There are several ways to demonstrate the "shell-growth" mechanism. Figure 5 shows (red line) the number of A12 particles in the largest A12-cluster as a function of time. The location of the largest crystal nucleus before, at, and after the onset of crystallization is determined from the positions of the A12 particles in the cluster. Before the onset of crystallization there is an ensemble of isolated A12 particles and small clusters of them up to sizes with 10. They appear well inside the droplet, typically at distance 5-10 $\sigma_{\mathrm{AA}}$ from the center of mass of the droplet. The small A12-clusters are not stable, and for this reason the location of the largest A12-cluster fluctuates with time. At the onset of crystallization, one of these clusters begins to grow rapidly. Figure 5 shows a representative example. The distance of the critical crystal nucleus of connected A12 particles to the center of the droplet is approximately ten molecular diameters.

The crystal nucleus contains more A particles than the connected A12 particles, namely, all the A particles that are not A12 


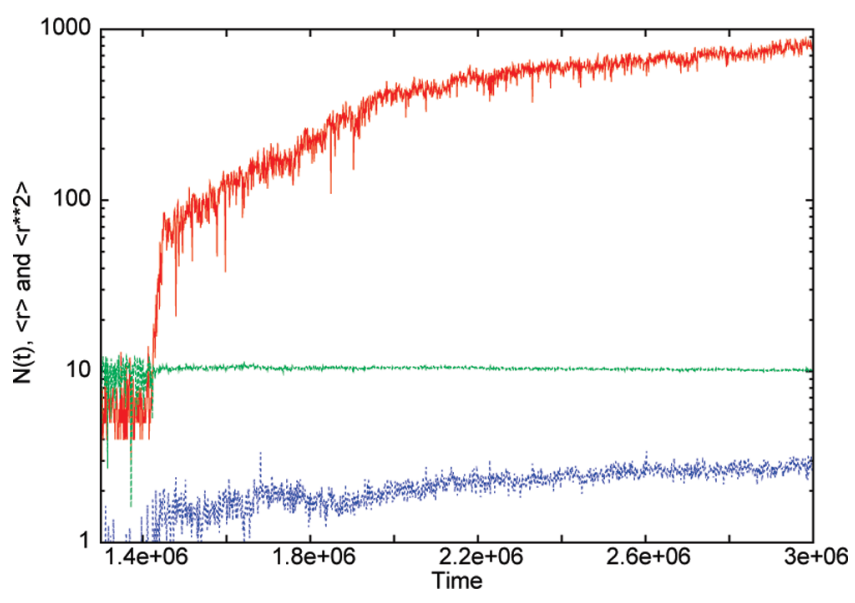

Figure 5. Number of A12 particles in the biggest A12-cluster, $N(t)$ (red line). The green line shows the mean distance $(\langle r(t)\rangle)$ of these A12 particles from the center of the droplet and the variance of $r(t)$ (blue) as functions of time. (A time step is $6.4 \times 10^{-13} \mathrm{~s}$. Notice the logarithmic ordinate plot by which all three functions can be plotted in one figure). The onset of crystallization appears after $1.43 \times 10^{6}$ time steps, where $N(t)$ increases dramatically. The crystal shell grows at a distance $\langle r(t)\rangle \approx 10$ molecular distances from the center of the droplet. The rather constant and small variance $(\approx 2$ diameters) of $r(t)$ demonstrates the shell-like growth.

Table 1. Mean Nucleation Time $\tau$ and the Mean Nucleation Distance $r_{n}$ from the Center of the Droplet for Different Molecular Fractions, $x_{\mathrm{B}}$, Corresponding to a Molal Concentration of Sulfuric Acid $m\left(\mathrm{H}_{2} \mathrm{SO}_{4} \mathrm{~mol} / \mathrm{kg}\right.$ of the Solute Molecules (Averaged over 25 Independent Crystallizations for Each Concentration) $)^{a}$

\begin{tabular}{lccr}
\multicolumn{1}{c}{$x_{\mathrm{B}}$} & $m\left(\mathrm{H}_{2} \mathrm{SO}_{4}\right)$ & $\tau / \tau^{*}$ & $r_{n} / \sigma_{\mathrm{AA}}$ \\
0.01 & 0.561 & $2.25 \times 10^{2}$ & $8.3 \pm 1.9$ \\
0.015 & 0.845 & $8.57 \times 10^{2}$ & $8.4 \pm 2.0$ \\
0.02 & 1.133 & $4.45 \times 10^{3}$ & $9.8 \pm 1.2$ \\
0.0225 & 1.252 & $1.97 \times 10^{4}$ & $10.4 \pm 1.1$ \\
${ }^{a}$ Time unit $\tau^{*}=\sigma_{\mathrm{AA}}\left(m_{\mathrm{A}} / \varepsilon_{\mathrm{AA}}\right)^{1 / 2} \approx 0.64 \mathrm{ps}$. & \\
\hline
\end{tabular}

particles but bounded to one of the A12 particles in the A12cluster. In a test calculation, we have included these molecules in the crystal cluster. Including these A particles located at the surface of the crystal nucleus, however, only changes the variance, not the mean distance $\langle r\rangle$. This is due to to the shell-like structure of the crystal with the center of the shell $\langle r\rangle$ one to two layers beneath the surface (see Tabel 1) and with an approximately equal amount of A particles attached to the crystal shell at the outer and inner surface of the shell.

The onset of crystallization in the example shown in Figure 5 took place after $1.43 \times 10^{6}$ time steps, where the number of connected A12 particles increased dramatically from $\approx 5-10$ to $\approx 70$ over a few thousand time steps. Thereafter, the crystal nucleus grew monotonically. The mean distance (green line) and variance (blue line) remained remarkably constant over the period of time in which the critical nucleus grew to $\approx 1000$ connected A12 particles (red line). The thickness of the crystal shell of A12 particles and their 12 nearest neighbors is of the order 3-4 layers.

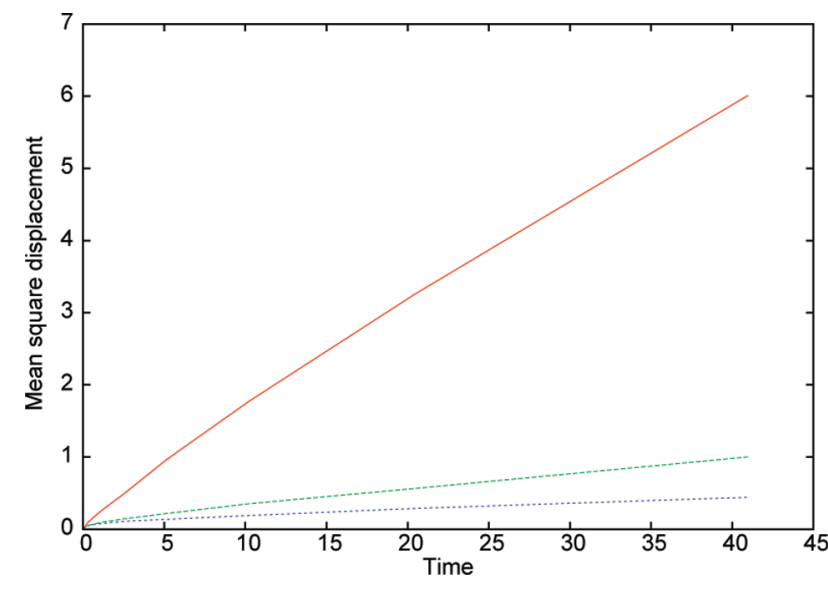

Figure 6. Mean-square displacement as a function of time. Red shows the A particles in the pure solvent sphere, $r>12$, which covers the droplet of a uniform mixture of A and B particles. Green dashes are the corresponding mean-square displacement for the A particles, which were in a shell with a distance to the center in the interval, $r \in[8,12]$, where the crystal nucleation appears. Blue dashes are the mean-square displacement for particles originally in the inner sphere with $r \leq 8$.

The onset of crystallization in droplets of 10000 particles was determined for the following solute mole fractions: $x_{\mathrm{B}}=0.01$, $0.015,0.02$, and 0.0225 , for $k_{\mathrm{B}} T / \varepsilon_{\mathrm{AA}}=0.40$ (corresponding to $180 \mathrm{~K}$ for an sulfuric acid aerosol $)$ and $\Delta=1.5\left(\Delta_{\text {mix }} H=-3.5 \mathrm{~kJ} / \mathrm{mol}\right)$. Data for the mean locations of the critical nuclei and mean crystallization times are given in Table 1.

The mean crystallization time, $\tau$, increases exponentially with the molecular fraction of the solute and thus with the strength of the exothermic mixture. This is consistent with the theory for crystallization in exothermic mixtures. ${ }^{10}$ Independent of where and how the crystal of the solvent nucleates, this behavior implies that the concentration of the exothermic solute controls the onset of crystallization consistent with experimental data for crystallization in aerosols. ${ }^{15}$ From the table it can be seen that as the solute concentration increases the location of the critical nucleus moves toward the interior interface at a distance $r \approx 12 \sigma_{\mathrm{AA}}$ from the center of the droplet (see Figure 2).

A priori, one might guess that with a thin surface wetted with the solvent the nucleation should start there. We never observed this, however, nor that nucleation began in the deep interior of the droplet. It always appeared 2-4 layers beneath the surface. The solvent particles in the (diluted) interface of the droplet have a high mobility (Figure 6), but the mobility (before onset of crystallization) is much less in the bulk mixture where the solvent particles are attached to the heavy and big solute B particles. There is, however, a significant difference in mobility between the diffusion in the zone where the crystallization initiates and the diffusion deep inside in the droplet. Figure 6 shows the rootmean-square (rms) displacement of the solvent A particles as a function of time. The diffusion in the zone where the onset of crystallization appears (green dashes) is twice of what it is in the interior of the droplet (blue dashes).

The vapor pressure of the solvent was determined before onset of crystallization in the supercooled nanodroplets as well as for a frozen droplet. The vapor pressure decreased roughly $40 \%$ upon crystallization, in qualitative agreement with experimental observations of the change in vapor pressure at ice formation caused by aerosols in the stratosphere. ${ }^{40}$ This implies that the 
nanodroplets after onset of nucleation may grow by swelling A particles from surrounding vapor, a fact that could be significant for understanding how ice production in stratospheric clouds takes place (see Section V).

Finally, we checked that the observed behavior does not depend on whether simulations are performed at constant temperature or at constant energy (i.e., with or without a thermostat). The vapor pressure is almost zero at the relevant degree of supercooling, implying that the temperature equilibration of a freezing supercooled nanodroplet must be slow. This implies that the (fast) crystallization of the droplet must take place at constant energy with the release of the latent heat at crystallization and an increase of the temperature in the droplet. For this reason we repeated some of the simulations but switched off the thermostat at the onset of crystallization. The result was that the temperature increased slowly during the crystallization, but with no qualitative differences in the shell-growth mechanism.

\section{SUMMARY}

The aim of the above MD investigations has been to determine the role of a strong exothermic solute on the onset and crystal growth in a supercooled droplet. All previous computer simulations ${ }^{2-5}$ of crystallization in systems with a free surface show that the onset of crystallization appears near the surface. The models studied include molecules with water-like tetrahedral ${ }^{4,5}$ and octahedral ${ }^{2,3}$ structure. The mixture with tetrahedral structure with strong hydrogen binding-like attractions ${ }^{4}$ resulted in an onset of crystallization, neither exactly at the surface nor in the interior of the system, in agreement with our observations. The KABLJ model for a supercooled mixture shows that the exothermic effect forces the onset of crystallization to take place not exactly at the surface but a few molecular layers beneath. In summary: simulations of quite different models all show the same qualitative behavior.

The tendency that the critical crystal nucleus appears a few molecular layers from the surface where the density equals the bulk density might be caused by the increased mobility of the molecules near the surface, ${ }^{41}$ including diffusion of $\mathrm{H}_{2} \mathrm{O}$ in water droplets. ${ }^{42}$ If so, it is the general phenomenon for crystallization of supercooled systems with a free surface, including crystallization of molecules with hydrogen bonds. In a highly viscous supercooled fluid, the flux of particles into the (critical) nucleus decreases exponentially with temperature and becomes an important factor for the rate of nucleation and growth. ${ }^{10}$ In the case of the KABLJ exothermic mixture, the mobility in the zone where the crystallization initiates is twice as big as in the interior of the droplet (see Figure 6). A slow diffusion suppresses the necessary concentration fluctuations, ${ }^{10}$ and this exothermic effect on the mobility forces the crystallization in the KABLJ droplets to take place a few molecular layers beneath the surface.

Perspective for Ice Nucleation in Aerosols. The present model for sulfuric acid aerosols is a simplification of an aerosol where the strong exothermic property of the mixture is maintained. We demonstrated above, in accordance with available experimental data for crystallization in aerosols, ${ }^{15}$ that the main qualitative property which determines crystallization in the droplets is the concentration of the solute, which determines the activity of solvent molecules. Our simulation data agree well with experimental data for crystallization of ice in aerosols. Thus, crystallization is only observed for small concentrations of the solute (sulfuric acid) and at supercooling corresponding to $\approx 180-190 \mathrm{~K}$ in agreement with data for ice formation in sulfuric acid aerosols. ${ }^{30}$ The strong exothermic mixing is caused by rearranging of hydrogen bonds between the water molecules and water molecules attached to the sulfuric acid molecules. The actual (atomic) structure within the aerosol of water and the sulfuric ions is, however, only of indirect importance. The onset of crystallization is controlled by the activity of water within the aerosol which determines the thermodynamic condition for ice crystallization, but it requires a detailed model if one aims for quantitative accuracy; such modeling should include the expansion of the solvent by freezing.

A small supercooled aerosol with a concentration of sulfuric acid of more than $2-3 \%$ is stable according to experiment ${ }^{43}$ and to our calculations. At decreasing temperatures it swells by absorbing $\mathrm{H}_{2} \mathrm{O}(\mathrm{g})$ from the air, sooner or later it crystallizes near the surface, and the interior of the droplet will be covered with a shell of ice. Missing from the model is the expansion of water by freezing. This unique quality of water results in a droplet with a fragile ice shell; if broken and removed, the inner highly concentrated droplet can act again as a template for further absorption of $\mathrm{H}_{2} \mathrm{O}(\mathrm{g})$ and thus catalyze continuous formation of ice crystals in the atmosphere. Ejected ice fragments would grow rapidly to bigger ice crystals and thereby bring the water vapor into equilibrium with ice, resulting in a mixture of ice crystals and liquid supercooled sulfate aerosols. Upon subsequent heating the ice crystals would evaporate, leaving behind the liquid aerosols. This resembles what has been observed in the upper troposphere and lower stratosphere (UTLS) during fast changing temperature conditions. ${ }^{16}$ If the temperature changes more slowly, heterogeneous nucleation involving solid impurities ${ }^{44,45}$ or organic material ${ }^{45,46}$ in the aerosol also plays an important role for solid particle formation in the UTLS.

\section{AUTHOR INFORMATION}

\section{Corresponding Author}

*E-mail: st@ruc.dk. Fax: (+) 454674 3020. Phone: (+) 454674 2062.

\section{ACKNOWLEDGMENT}

The centre for viscous liquid dynamics "Glass and Time" is sponsored by the Danish National Research Foundation (DNRF).

\section{REFERENCES}

(1) Baletto, F.; Ferrando, R. Rev. Mod. Phys. 2005, 77, 371

(2) Chushak, Y. G.; Bartell, L. S. J. Phys. Chem. B 1999, 103, 11196.

(3) Chushak, Y. G.; Bartell, L. S. J. Phys. Chem. A 2000, 104, 9328.

(4) Li, T.; Donadio, D.; Ghiringhelli, L. M.; Galli, G. Nat. Mater. 2009, 8, 726.

(5) Zasetsky, A. Y.; Remorov, R.; Svishchev, I. M. Chem. Phys. Lett. 2007, 435, 50.

(6) Djikaev, Y. S.; Tabazadeh, A.; Hamill, P.; Reiss, H. J. Phys. Chem. A 2002, 106, 10247.

(7) Djikaev, Y. S.; Tabazadeh, A.; Reiss, H. J. Chem. Phys. 2003, 118,6572 .

(8) Djikaev, Y. S. J. Phys. Chem. A 2008, 112, 6592.

(9) Kob, W.; Andersen, H. C. Phys. Rev. E 1993, 51, 4626. ibid 1995, $52,4134$.

(10) Toxvaerd, S.; Pedersen, U. R.; Schrøder, T.; Dyre, J. C. J. Chem. Phys. 2009, 130, 224501. 
(11) Rowlinson, J. S. Liquid and Liquid Mixtures; Butterworths: London, 1969.

(12) Solomon, S.; Qin, D.; Manning, M.; Chen, Z.; Marquis, M.; Averyt, K. B.; Tignor, M.; Miller, H. L. IPCC Climate Change 2007: The Physical Science Basis. Contribution of Working Group I to the Fourth Assessment Report of the Intergovernmental Panel on Climate Change; Cambridge Univ. Press Cambridge: UK, 2007.

(13) Fueglistaler, S.; Dessler, A. E.; Dunkerton, T. J.; Folkins, I.; Fu, Q.; Mote, P. W. Rev. Geophys. 2009, 47, RG1004.

(14) World Meteorological Organization (WMO), Scientific Assessment of Ozone Depletion: 2006, Global Ozone Res. and Monitoring ProjectReport No. 50, Geneva, 2007.

(15) Koop, T; Luo, B.; Tsias, A.; Peter, T. Nature 2000, 406, 611.

(16) Carslaw, K. S.; Wirth, M.; Tsias, A.; Luo, B. P.; Dörnback, A.; Leuthbecher, M.; Volkert, H.; Renger, W.; Bacmeister, J. T.; Peter, T. J. Geophys. Res. 1998, 103, 5785.

(17) J. Schreiner, J.; Voigt, C.; Kohlmann, A.; Arnold, F.; Mauersberger, K.; Larsen, N. Science 1999, 283, 968.

(18) Voigt, C.; Schreiner, J.; Kohlmann, A.; Zink, P.; Mauersberger, K.; Larsen, N.; Deshler, T.; Kröger, C.; Rosen, J.; Adriani, A.; Cairo, F.; Di Donfrancesco, G.; Viterbini, M.; Ovarlez, J.; Ovarlez, H.; David, C.; A. Dörnbrack, A Science 2000, 290, 1756.

(19) Beyer, K. D; Hansen, A. R.; Poston, M J. Phys. Chem. A 2003, 107,2025 .

(20) Gable, C. M.; Betz, H. F.; Maron, S. H. J. Am. Chem. Soc. 1950, $72,1445$.

(21) Giauque, W. F.; Hornung, E. W.; Kunzler, J. E.; Rubin, T. F. J. Am. Chem. Soc. 1960, 82, 62.

(22) Walrafen, G. E.; Yang, W.-H.; Chu, Y. C.; Hokmabadi, M. S. J. Solution Chem. 2000, 10, 905.

(23) Walrafen, G. E.; Yang, W.-H.; Chu, Y. C. J. Phys. Chem. A 2002, 106, 10162.

(24) Beyer, K. D.; Hansen, A. R.; Raddatz, N. J. Solution Chem. 2000, 29, 905.

(25) Coulson, C. A. Nature 1969, 221, 1106.

(26) Giauque, W. F.; Kunzler, J. E.; Hornung, E. W. J. Am. Chem. Soc. 1956, 78, 5482 .

(27) Toxvaerd, S. J. Phys. Chem. C 2007, 111, 15620.

(28) Myhre, C. E. L.; Nielsen, C. J.; Saastad, O. W. J. Chem. Eng. Data 1998, 43, 617.

(29) Gmitro, J. I.; Vermeulen, T. AIChE J. 1964, 10, 740.

(30) Murray, B. J.; Knopf, D. A.; Bertram, A. K. Nature 2005, 434, 202.

(31) Toxvaerd, S. J. Chem. Phys. 2002, 117, 10303.

(32) For MD details see: Toxvaerd, S. Mol. Phys. 1991, 72, 159.

(33) The routine sorts the particle by using a the nearest-neighbour list during a MD simulation. The routine is not published but will be given by request to the authors.

(34) Toxvaerd, S. J. Chem. Phys. 1971, 55, 3116.

(35) Barker, J. A.; Henderson, D.; Abraham, F. F. Physica 1981, 106A, 226.

(36) Bishop, C. L.; Pan, D; Liu, L. M.; Tribello, G. A.; Michaelides, A.; Wang, E. G.; Slater, B. Faraday Discuss. 2009, 141, 277.

(37) ten Wolde, P. R.; Frenkel, D. J. Chem. Phys. 1998, 109, 9901.

(38) Toxvaerd, S. J. Chem. Phys. 2001, 115, 8913.

(39) Velasco, E.; Toxvaerd, S. Phys. Rev. Lett. 1993, 71, 388.

(40) Larsen, N.; Knudsen, B. M.; Voigt, C.; Weisser, C.; Kohlmann, A.; Schreiner, J.; Mauersberger, K.; Deshler, T.; Kröger, C.; Rosen, J. M.; Kjome, N. T.; Adriani, A.; Cairo, F.; Di Donfrancesco, G.; Ovarlez, J.; Ovarlez, H.; Dörnbrack, A.; Birner, T. J. Geophys. Res. 2002, 107, 1029.

(41) SeeD. Duque, D; P. Tarazona, P.; E. Chacón, E. J. Chem. Phys. 2008, 128, 134704 and the references therein.

(42) Malenkov, G. G. Colloid J. 2010, 72, 653.

(43) Koop, T; Luo, B.; Biermann, U. M.; Crutzen, P. J.; Peter, T. J. Phys. Chem. A 1997, 101, 1117.

(44) Drdla, K.; Browell, E. V. J. Geophys. Res. 2004, 109, D10201.

(45) Murphy, D. M.; Thomson, D. S.; Mahoney, M. J. Science 1998, 282,1664 .
(46) Froyd, K. D.; Murphy, D. M.; Lawson, P.; Baumgardner, D.; Herman, R. L. Atmos. Chem. Phys. Discuss. 2009, 9, 20347. 